\title{
A novel multiscale neutron-diffraction-based experimental approach for granular media
}

\author{
S. D. ATHANASOPOULOS*, S. A. HALL †t and J. F. KELLEHER
}

\begin{abstract}
The complexity of granular (geo-)materials is associated with the mobility and interaction of their constituent particles. Under the effect of loading, these materials exhibit a highly inhomogeneous behaviour, which might vary significantly as the loading develops (i.e. certain grain assemblies take up the load whilst other, neighbouring assemblies fall into states of lower load). To that end, understanding the (micro-)mechanisms related to the distribution and evolution of forces/stresses through granular media requires appropriate, spatially resolved measurements. Neutron strain scanning (NSS) is an experimental technique based on diffraction measurements that has been successfully used in recent years to infer force/stress distribution in granular materials under load, by measuring the crystallographic strains of grains. In this work, first results from a new experimental approach involving simultaneous NSS and digital image correlation (DIC) of quartz sand under load in a specially designed plane-strain apparatus are presented. The combined use of these techniques allows the investigation of deformation mechanisms in granular media, such as sand, at different scales. Therefore, a completely novel multiscale analysis of granular (geo-)materials can be made that is, associating the traditional macroscale measurements with the mesoscale characterisation of the strain field (through DIC) and the inferred microscale stress distribution (through NSS).
\end{abstract}

KEYWORDS: in situ testing; particle-scale behaviour; strain; stress analysis

Published with permission by the ICE under the CC-BY 4.0 license. (http://creativecommons.org/licenses/by/4.0/)

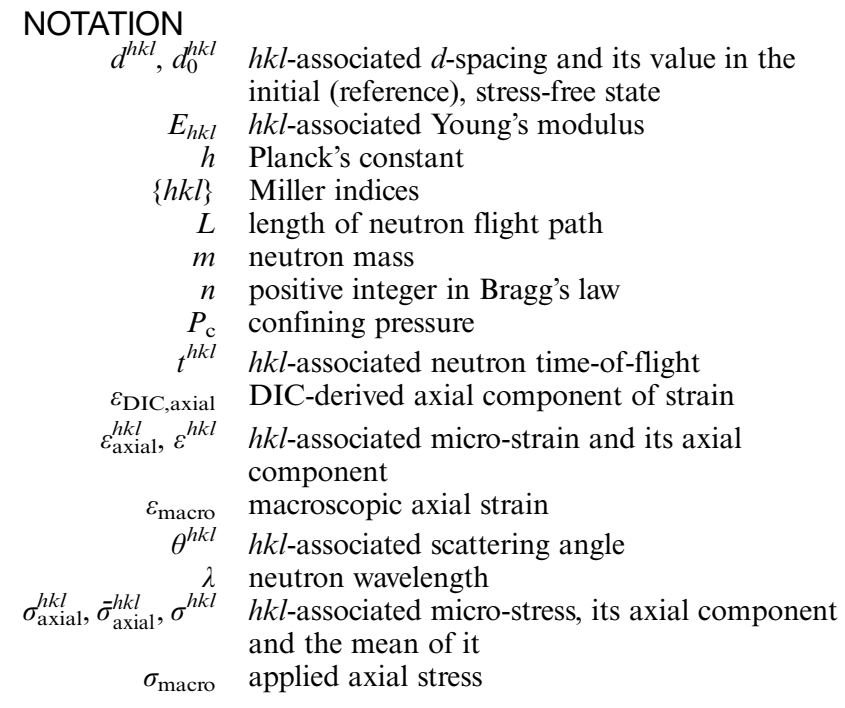

\section{INTRODUCTION}

The vast growth of full-field measurement techniques over the past few decades (see Viggiani \& Hall, 2008 and

Manuscript received 7 December 2018; first decision 24 March 2019; accepted 24 March 2019.

Published online at www.geotechniqueletters.com on 3 October 2019.

*Division of Solid Mechanics, Lund University, Lund, Sweden (Orcid:0000-0002-2638-4802).

$†$ Division of Solid Mechanics, Lund University, Lund, Sweden.

tLund Institute of Advanced Neutron and X-Ray Science, Lund, Sweden.

§ISIS Neutron Source, Rutherford Appleton Laboratory, Harwell Oxford, Didcot, UK. references therein for an overview) has enabled the investigation of the - inherently multiscale - behaviour of geomaterials at different scales. For granular (geo-)materials, in particular, various techniques have been employed towards the understanding of their complexity (e.g. Charalampidou et al., 2011; Desrues \& Andò, 2015), which is manifested by the mobility and interaction of their constituent particles. Under the effect of loading, and as deformation develops, these materials exhibit a highly inhomogeneous mechanical behaviour, which might vary significantly throughout the granular skeleton, as it is related to the evolution of force/stress distribution and requires appropriate, spatially resolved measurements.

Since the first photoelasticity based experimental works on force transmission through glass particles in the 1950s (e.g. Wakabayashi, 1950), there have been several studies, using various methods, in the effort of characterising the stress distribution in granular (geo-)materials (e.g. Saadatfar et al., 2012; Hurley et al., 2014). Neutron strain scanning (NSS) is a diffraction based technique that has been successfully used to infer the force/stress distribution in granular media under load (e.g. Hall et al., 2011; Wensrich et al., 2012), by measuring the crystallographic strains of grains.

This letter introduces a new experimental approach, which involves the simultaneous application of NSS and digital image correlation (DIC) on granular geomaterials under load, using a novel plane-strain apparatus. Herein, a few selected results from the first NSS-DIC experiment on quartz sand are presented, aiming to highlight the potential of the suggested experimental approach, which is still under development. Subsequently, the discussion focuses mainly on the identification of the information that can be extracted from the NSS data, as well as on how this microscale information can be correlated to other measurements, at different scales (i.e. the macroscale boundary measurements and the mesoscale DIC-derived strain field). 


\section{EXPERIMENTAL APPROACH}

\section{Basic principles of neutron diffraction}

NSS is a neutron diffraction technique able to provide information on the distribution of strain and, therefore, stress deep inside bulk crystalline structures (see Hutchings et al., 2005 for a thorough description). Strain measurement by NSS relies on the fact that the interplanar spacing of crystallographic planes (i.e. the $d$-spacing) may serve as a strain gauge embedded in crystalline matter. Neutron diffraction can be described through Bragg's law, a relationship between the neutron wavelength, $\lambda$, the $d$-spacing of a certain $\{h k l\}$ family of crystallographic planes ( $h k l$ being the Miller indices), $d^{h k l}$, and the angle at which the neutrons are scattered coherently and elastically by the corresponding planes, $\theta^{h k l}$,

$$
n \lambda=2 d^{h k l} \sin \theta^{h k l}
$$

where $n$ is a positive integer. When Bragg's law is fulfilled, a reflection (i.e. a Bragg peak), corresponding to a specific $h k l$ orientation, can be identified in a diffraction pattern and shifts in its position are directly related to elastic changes in $d$-spacing (i.e. elastic crystallographic - or grain - strains) of the respective planes. Other characteristics of a Bragg peak can provide further information on a material's microstructure (e.g. subgrain plastic deformations, such as dislocations, can be attributed to peak width variations), but in the present work only peak position shifts are considered, which are associated with the elastic component of strain.

For polycrystalline materials, such as sand, it is assumed that within a sub-volume of a specimen illuminated by neutrons (i.e. the gauge volume - GV), typically of the order of a few $\mathrm{mm}^{3}$, there exists a substantial subset of scattering grains with a specific $h k l$ orientation that fulfils Bragg's law (i.e. the scatterers). Applying a load to a specimen would result in the Bragg-peak position of the scatterers to shift, due to the strain all the constituent grains of a GV would experience. Hence, a mean, elastic, $h k l$-associated microstrain, $\varepsilon^{h k l}$, can be calculated by

$$
\varepsilon^{h k l}=\frac{d^{h k l}-d_{0}^{h k l}}{d_{0}^{h k l}}
$$

where $d_{0}^{h k l}$ is a reference, stress-free value. $\varepsilon^{h k l}$ is assumed to be representative of the entire bulk of the grains in the GV (i.e. voids are excluded) and its orientation is defined by the scattering $\boldsymbol{Q}$-vector that bisects the incident and the diffracted neutron beams. As the measured crystal-lattice strains are purely elastic, regardless of any plasticity the specimen might be experiencing macroscopically, a mean, $h k l$-associated micro-stress, $\sigma^{h k l}$, can be calculated through Hooke's law and the respective, also $h k l$-associated, elastic constants.

\section{Experimental method}

The experimental set-up consists of a specially designed plane-strain apparatus (Fig. 1) that can accommodate prismatic specimens of $60 \times 30 \times 20 \mathrm{~mm}^{3}$ (height $\times$ width $\times$ thickness), an image acquisition system (Fig. 2) and a pressure controller. Here, only the apparatus' loading conditions are outlined, whilst a more detailed description can be found in Athanasopoulos et al. (2018) and in future publications. Plane-strain conditions are fulfilled by applying a force along the longitudinal axis of the specimen (Fig. 1a) and deformation being limited to develop in only one of the other two directions (i.e. $x$-axis), through the combination of a pair of deformable, pressure-controlled

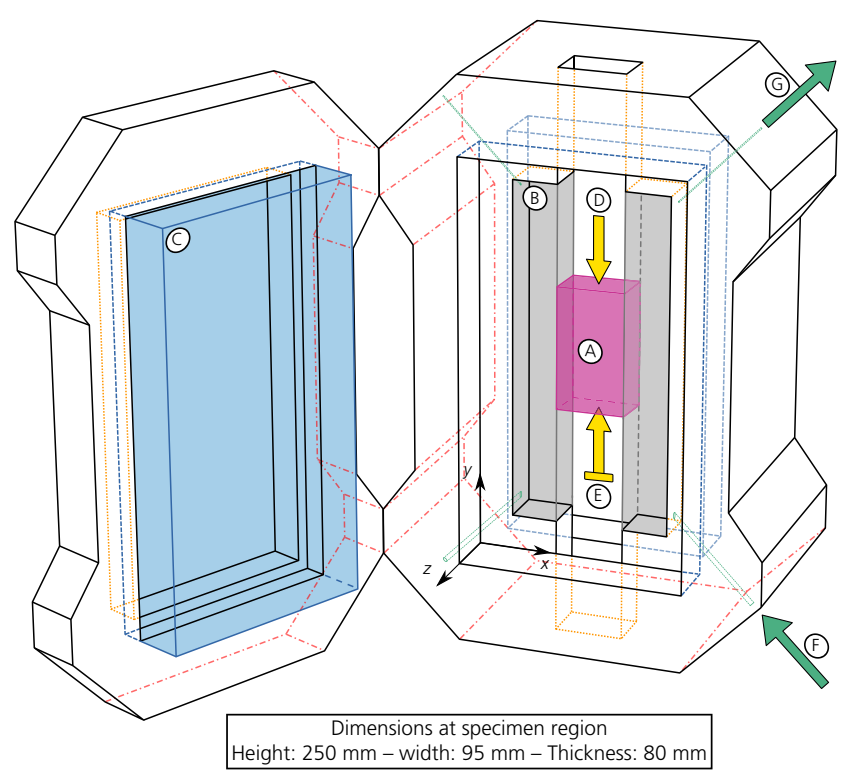

Fig. 1. Schematic representation of the plane-strain loading apparatus. (a) Specimen, (b) pressure-controlled cushions, (c) sapphire platens, (d) moving piston, (e) fixed piston, (f) pressure liquid supply and (g) air escape

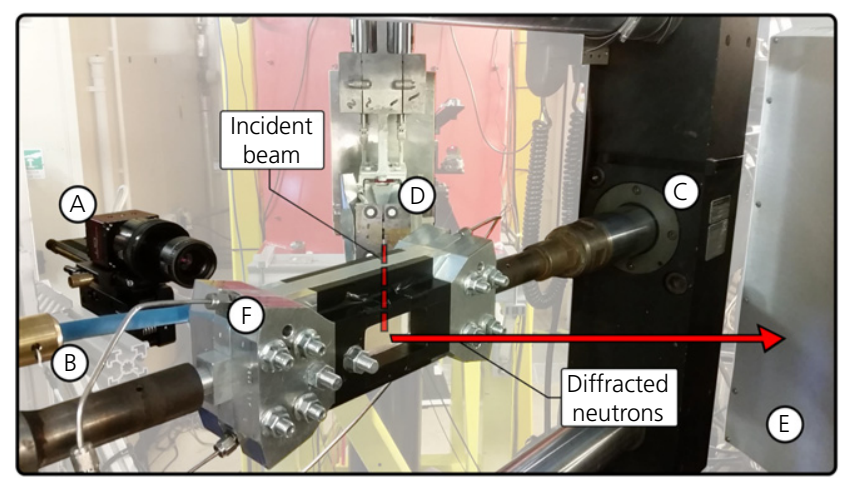

Fig. 2. The experimental set-up in the ENGIN-X neutron strain scanner at the ISIS spallation source in the UK. (a) High resolution (28.8 MP) Prosilica GT 6600 camera, (b) custommade LED lighting system, (c) Instron stress rig (100 kN), (d) beam defining optics system, (e) detector, (f) connection of the apparatus to a 3 MPa GDS Standard Pressure Controller

cushions (Fig. 1b) and a pair of sapphire platens (Fig. 1c), which prevent any deformation in the third direction. Minimisation of friction between both the specimen and the cushions against the sapphire platens is accomplished by lubrication with fluorinated grease.

Figure 2 shows the experimental set-up with the apparatus mounted in a stress rig (Fig. 2c) at the ENGIN-X neutron strain scanner (Santisteban et al., 2006). ENGIN-X is an energy-dispersive - or time-of-flight (TOF) - diffractometer, as it uses a polychromatic (i.e. wide wavelength) neutron beam dispersing in relation to its TOF from the source. Diffracted neutrons are recorded as a function of their TOF, $t$, at a fixed $2 \theta$ angle and Bragg's law can be satisfied for multiple wavelengths and $h \mathrm{kl}$ orientations. Thus, a TOF diffractogram contains multiple reflections, each associated with a different subset of scatterers inside a GV. A mean micro-strain of a GV can, therefore, be determined through Bragg's law from the peak shift of each $h k l$ reflection in the 


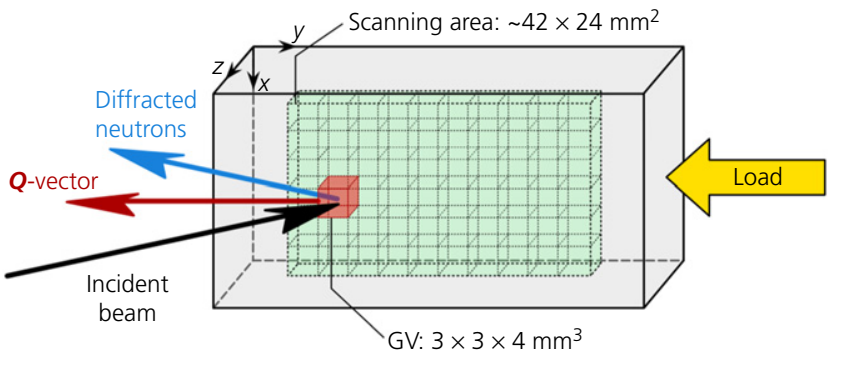

Fig. 3. Schematic representation of the measurement set-up in the specimen

TOF spectrum, by making use of

$$
\lambda=\frac{t^{h k l} h}{m L}
$$

where $h$ is Planck's constant, $m$ is the neutron mass and $L$ is the length of the neutron flight path.

The experiment discussed in the following involved plane-strain loading of Fontainebleau NE34 quartz sand $\left(D_{50}: 210 \mu \mathrm{m}\right)$ at a confining pressure, $P_{\mathrm{c}}$, of $3 \mathrm{MPa}$. It is noted that one of the reasons for reaching such an elevated pressure was to ensure, in this developmental stage of the method, a strong peak-shift signal-to-noise ratio in the recorded diffractograms. NSS measurements were realised at a fixed $2 \theta$ angle of $90^{\circ}$, whilst the loading system was positioned at $45^{\circ}$ to the incoming beam, giving a direction of strain measurement (i.e. of the $\boldsymbol{Q}$-vector) parallel to the applied loading axis (Fig. 3).

The first stage of the loading involved attaining the confining pressure of $3 \mathrm{MPa}$ with simultaneous increase of the axial stress, $\sigma_{\text {macro, }}$ to maintain isotropic in-plane pressure boundary conditions. Subsequently, the specimen was loaded over a series of load steps (LSs), reaching a maximum $\sigma_{\text {macro }}$ of approximately $19 \mathrm{MPa}$ (Fig. 4(a)). At each LS, the loading was paused, with the axial displacement and $P_{\mathrm{c}}$ held constant, to acquire diffraction data over a two-dimensional grid of $6 \times 10 \mathrm{GVs}$ in the middle of the specimen (Fig. 3). The scanning coverage of the specimen in the $x y$ plane was approximately $56 \%$ (of the initial dimensions of the specimen), using a $3 \times 3 \times 4 \mathrm{~mm}^{3} \mathrm{GV}$ (Fig. 3), and the scanning time for each $\mathrm{GV}$ was about $4 \mathrm{~min}$. In parallel, photographs for the DIC were acquired every $5 \mathrm{~min}$ during the NSS measurements and every $6 \mathrm{~s}$ during the loading of the specimen. The choice of the scanning area and measurement time per GV was based on a trade-off between the ENGIN-X characteristics, the available beamtime and the quality of the acquired data.

\section{Data analysis}

The processing of the NSS data was realised with an in-house MATLAB ${ }^{\circledR}$ code and the DIC analysis was performed using VIC2D ${ }^{\circledR}$ from Correlated Solutions ${ }^{\circledR}$. Regarding the NSS data processing, multiple reflections, corresponding to different $h k l$ orientations, were recorded during the experiment, but only the peak corresponding to the $\{211\}$ family of crystallographic planes was analysed for the results presented herein (see doi.org/10.5286/ISIS.E.87816269 for the complete dataset). The diffraction peaks for this reflection were analysed by peak fitting, using the MATLAB ${ }^{\circledR}$ Curve Fitting Toolbox ${ }^{\mathrm{TM}}$ and an asymmetric Gaussian lineshape (Stancik \& Brauns, 2008) as the fitting function. The peak-fitting variables were the amplitude (i.e. the intensity of the signal), the centroid (i.e. the $d$-spacing value at the maximum intensity), the width, the background (i.e. the noise of the signal) and the asymmetry factor of the lineshape. The axial component of the micro-stress, $\sigma_{\text {axial }}^{211}$, was then derived from $\varepsilon_{\text {axial }}^{211}$, which was calculated by equation (2) from the fitting-defined $d$-spacing values, using a Young's modulus $E_{211}=82.44 \mathrm{GPa}$, but without taking into consideration the void ratio (see also the following section).

\section{RESULTS AND DISCUSSION}

Figure 4 shows the DIC-derived axial component of the total strain field, $\varepsilon_{\text {DIC,axial }}$, and the NSS-inferred total $\sigma_{\text {axial }}^{211}$ mappings, the original grid of which has been linearly interpolated onto a finer grid, along with both $\sigma_{\text {macro }}$ and the mean micro-stress for each LS, $\bar{\sigma}_{\text {axial }}^{211}$, as functions of the macroscopic axial strain, $\varepsilon_{\text {macro }}$ (henceforth, the macro- and micro-curve, respectively).

In Section I of the loading, the micro-curve follows, in principle, a similar trend to the macro-curve, apart from a drop-down between the first two LSs, followed by a steep re-gain of the load. The corresponding $\sigma_{\text {axial }}^{211}$ mappings exhibit significant spatial and temporal variations (i.e. load shifts), implying overall grain reorganisation, however, $\sigma_{\text {axial }}^{211}$ increases (i.e. the mappings get darker) with an increasing $\sigma_{\text {macro }}$. These successive shifts suggest that the micro-stress distribution evolves through unloading in one region of the specimen leading to another area taking up the load.

In Section II, the micro-curve continues to be in good agreement with the macro-curve, despite the fluctuations, until a maximum $\bar{\sigma}_{\text {axial }}^{211}$ is reached, which occurs before the maximum $\sigma_{\text {macro }}$. After attaining its maximum value (LS 8), a substantial relaxation is seen in $\bar{\sigma}_{\text {axial }}^{211}$. This drop could indicate a reduction in the specimen stress, but this would not be realistic. In fact, since the diffraction measurements were realised only along the axial direction, the micro-curve trend implies only that the component of the micro-stress aligned to the loading direction reached a maximum earlier than the peak stress measured externally. The subsequent drop in $\bar{\sigma}_{\text {axial }}^{211}$ can be interpreted as being related to rotation of the maximum micro-stress away from the axial direction (i.e. decreasing $\sigma_{\text {axial }}^{211}$ ), which might be associated with the formation and, eventually, slipping of an inclined localised deformation zone. The preceding fluctuations in $\bar{\sigma}_{\text {axial }}^{211}$ may be attributed to similar, smaller localised events that progressively contributed to the evolution of this localised zone. In conjunction with the $\bar{\sigma}_{\text {axial }}^{211}$ evolution, the micro-stress mappings indicate the development of a diagonal structure of higher compression, traversing the mappings from left to right, that becomes more evident and gradually thins with loading. It can also be seen that regions with more vertical features (i.e. with higher angle) of greater compression at a certain LS (e.g. top-middle in the LS 8 mapping) evolve into more inclined features at the consecutive LS (i.e. LS 9). This evolution of the localised deformation mechanisms, within what appears to be a shear zone, is consistent with the aforementioned observations regarding the micro-curve and stress rotation.

In Section III, both the micro- and macro-curve show post-peak plateaus, a trend likely related to the continued slipping of the shear zone and minimal changes in the stress state (i.e. as $\bar{\sigma}_{\text {axial }}^{211}$ does not change significantly, there is probably no rotation of the stress whilst the shear-band is slipping). Prior to the final plateau, the macro-curve shows a peak stress followed by a macroscopic relaxation, whilst $\bar{\sigma}_{\text {axial }}^{211}$ shows an increase. This, again, suggests a 


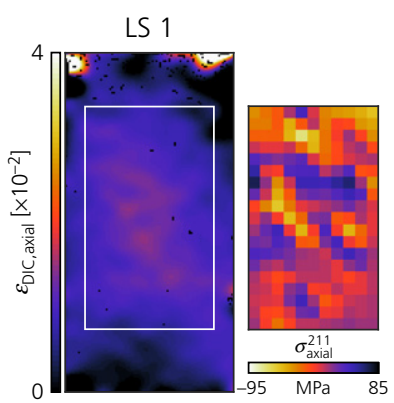

LS 5
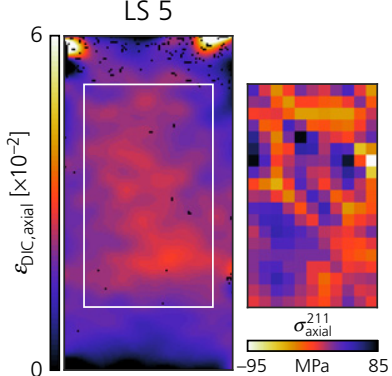

LS 9
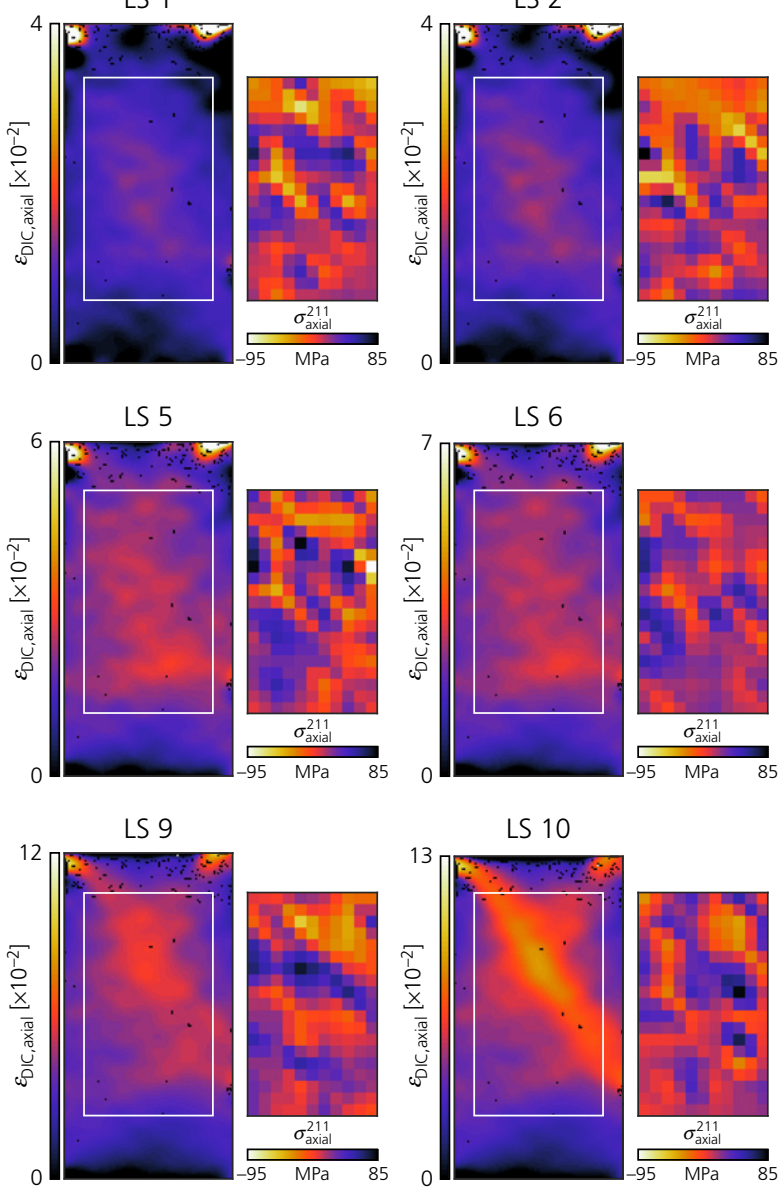

LS 6

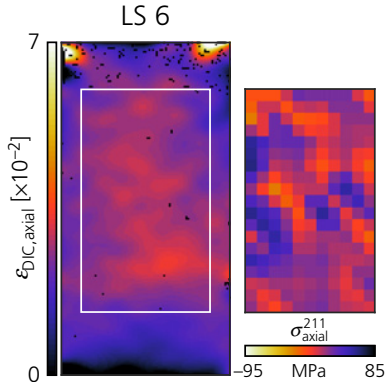

LS 10
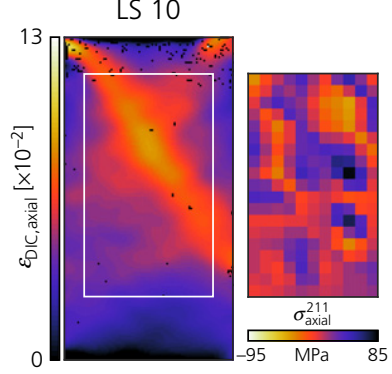

(b)
LS 3

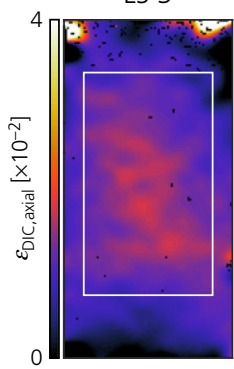

LS 7

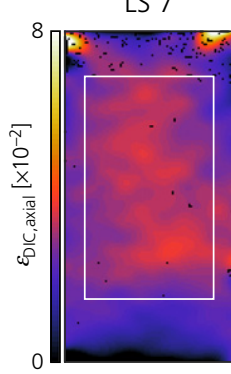

LS 11

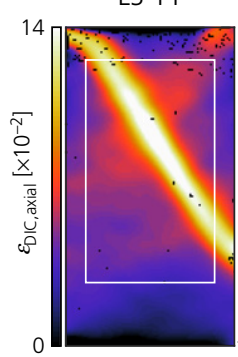

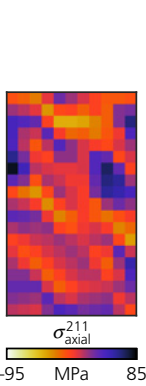

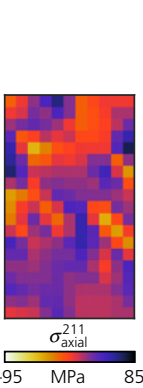

$-95 \mathrm{MPa} \quad 85$

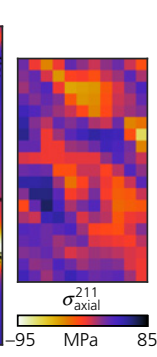

LS 4

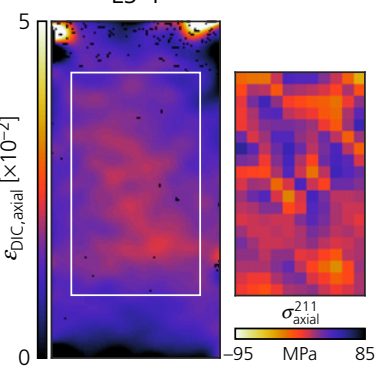

LS 8

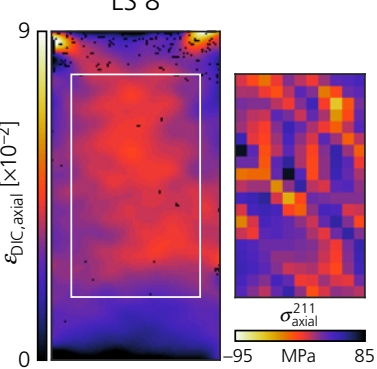

LS 12

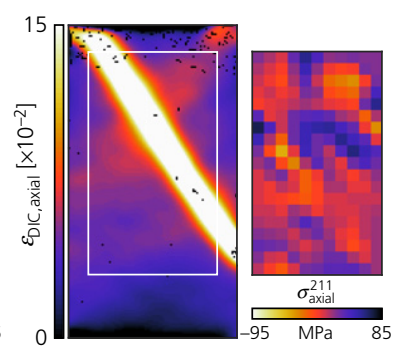

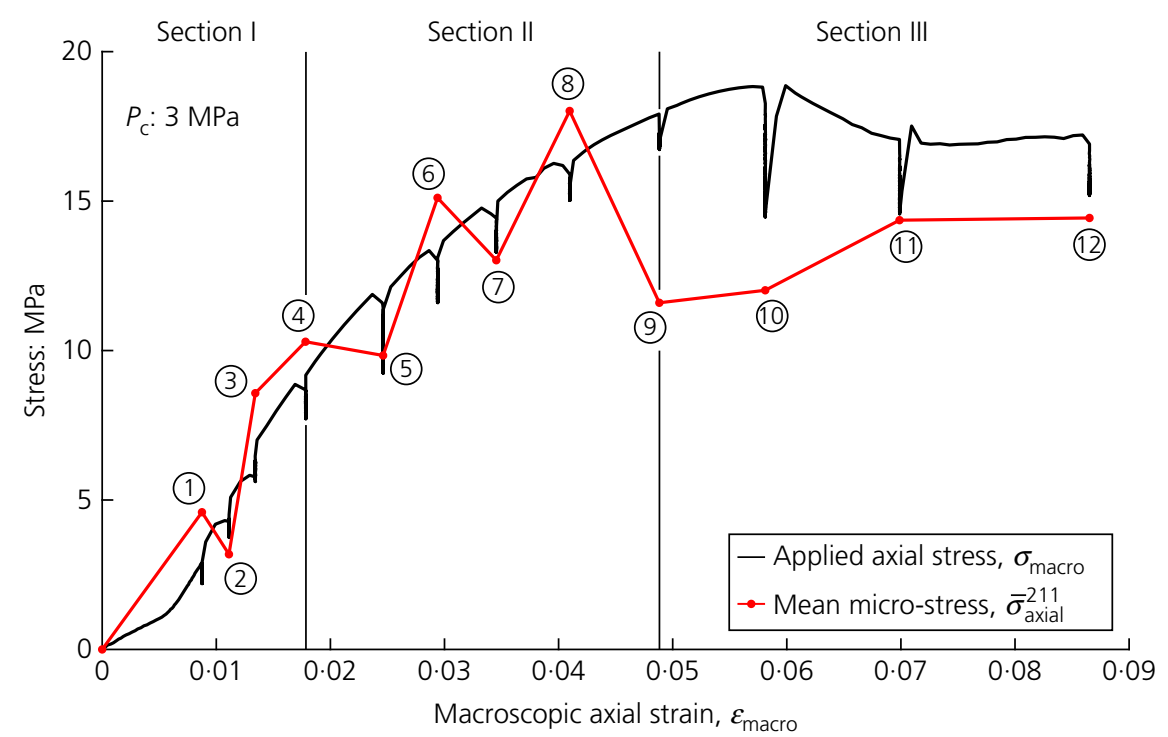

(a)

Fig. 4. (a) The applied axial stress and the mean axial component of the micro-stress as functions of the macroscopic axial strain. (b) (colour online) The DIC-derived axial component of the total strain field (left) and the NSS-derived mappings of the axial component of the total micro-stress (right) per load step

rotation of the stresses such that the axial micro-stress component increased, whilst the shear zone continued to evolve. The corresponding mappings exhibit the development of the diagonal structure. More specifically, and in line with the observations from the micro-curve, the mapping at the maximum $\sigma_{\text {macro }}$ has a slightly more vertical, but still diagonally pronounced structure of higher compression, than the mapping of the previous LS. The mapping at the beginning of the plateau shows a very clear diagonal feature that seems to contain another, horizontal one in the middle. The final mapping still shows the diagonal feature of high compression, together with the horizontal one, but this is less 
evident and is mainly to the middle-right of the mapping. The corresponding strain field from the DIC exhibits a strongly localised feature, which suggests that, with the further slipping of the shear zone, the stress is less localised than the strain. However, future analysis should investigate the incremental strain evolution, as this might provide more insight into the localisation of the active deformation, as opposed to the accumulated effects.

Regarding the comparison of the micro-stress mappings to the DIC results, a relatively good and, in certain cases, prominent correlation can be made. In particular, it is worth noticing the general spatial accordance of the high compression features throughout the mappings, spanning from the upper-left corner to the middle and bottom of the right-hand side, to the DIC-derived strain field evolution. A characteristic example is the transition from LS 8 (i.e. at the maximum $\bar{\sigma}_{\text {axial }}^{211}$ ) to LS 9 described above, where the more vertical features that evolve to more inclined, diagonal features, coincide - remarkably - with the development of the regions of localised strain into a more evident diagonal structure in the corresponding DIC results.

Key questions with any new experimental approach concern the resolution and possible sources of error or uncertainty. Regarding the former, crystallographic-strain resolution of the order of $10^{-5}$ is achievable at ENGIN-X, which has been verified by calibration tests on metals. The peak fitting of the NSS data in this work indicates a similar strain resolution (i.e. of the order of $10^{-5}$ ), which, after an error propagation analysis, equates to an uncertainty of approximately 4.5 MPa for the micro-stress measurements. This is a relatively high error if it is correlated to the micro-curve trend, but it is small compared to variations observed within the NSS mappings. Nonetheless, the trend in $\bar{\sigma}_{\text {axial }}^{211}$ is clear and, therefore, the propagated uncertainty is deemed to be an overestimate. This highlights the need for realising appropriate NSS calibration measurements for granular media, which, as of yet, do not exist and are, thus, a future objective.

It is noted that $\bar{\sigma}_{\text {axial }}^{211}$ is potentially underestimated due to the fact that the NSS mappings, from which it is determined, do not cover the full width of the specimen. Moreover, the difference between $\sigma_{\text {macro }}$ and $\bar{\sigma}_{\text {axial }}^{211}$, presented herein, could also be, in part, due to friction effects, in addition to the aspects discussed above. Furthermore, in the current analysis the porosity and its development have not been accounted for and, therefore, the micro-stress is overestimated. Whilst an estimate of the initial porosity might be made, the initial structure of the specimen in the studied experiment was not homogeneous. In particular, the specimen was produced with its axial direction vertical and, subsequently, it was placed - locked inside the apparatus - in the loading system with its axial direction horizontal. This resulted in some redistribution of the sand, possibly due to some cavity (at the upper-right corner of the specimen) that was not observed, producing the heterogeneity seen in both the DIC fields and NSS mappings of the early LSs. In future work, if a more homogeneous initial state is achieved, an assumption on the initial porosity field could be made and its development could be determined from the DIC data.

\section{CONCLUSIONS}

A new, neutron-diffraction-based experimental approach for studying granular media under load has been presented. By associating traditional macroscale boundary measurements with the mesoscale digital image correlation derived strain field and the neutron strain scanning inferred microscale stress distribution, a completely novel multiscale analysis for granular (geo-)materials has been enabled.
The main objective was to use spatially resolved NSS measurements, to map the evolution of grain strains under the effect of loading and thus, determine the stress distribution throughout the material, from a continuum point of view, and its evolution with - localised deformation. The correlation of structures in the NSS and DIC results indicate that the combination of the two techniques in a single experimental approach can provide novel insight into the coupled evolution of stress-strain distributions throughout granular media and how, eventually, material failure is reached. Further investigation is needed to understand the features that do not correlate between the DIC and NSS results, for example by considering incremental strains and more detailed analysis of the NSS data. However, since the two techniques measure different properties, complete structural agreement between the two should not be expected and these differences might reveal more information on the mechanics.

The micro-stress mappings and the micro-curve provide a quantitative characterisation of how the granular skeleton responds and have the potential to make a significant impact on the understanding of the (micro-) mechanical behaviour of granular media. To that end, future work will involve higher spatial and temporal resolution (i.e. larger, denser scanning grid and more load steps, respectively), as well as NSS measurements at more orientations (i.e. two more) for the complete determination of the in-plane stress tensor. In addition, due to the novelty of the experimental approach, further investigations are required to address the uncertainties and the resolution of the method.

\section{ACKNOWLEDGEMENTS}

The authors wish to thank Dr Jonas Engqvist, a research engineer in the Division of Solid Mechanics of Lund University in Lund, and Dr Tung Lik Lee, an ENGIN-X instrument scientist, for their contribution in the preparation and realisation of the experiment, respectively. This research had the financial support of the Swedish Research Council (Vetenskapsrådet, VR), grants No. 621-2012-4234 and No. 729 2015-04398. We are also grateful for the beamtime provided by ISIS, beamline ENGIN-X.

\section{REFERENCES}

Athanasopoulos, S. D., Hall, S. A., Kelleher, J. F., Pirling, T., Engqvist, J. \& Hektor, J. (2018). Mapping grain strains in sand under load using neutron diffraction scanning. In Micro to Macro mathematical modelling in soil mechanics, Trends in Mathematics (eds P. Giovine, P. Mariano, G. Mortara), pp. 23-33. Cham, Switzerland: Birkhäuser.

Charalampidou, E. M., Hall, S. A., Stanchits, S., Lewis, H. \& Viggiani, G. (2011). Characterization of shear and compaction bands in a porous sandstone deformed under triaxial compression. Tectonophysics 503, No. 1-2, 8-17.

Desrues, J. \& Andò, E. (2015). Strain localisation in granular media. Compt. Rendus Phys. 16, No. 1, 26-36.

Hall, S. A., Wright, J., Pirling, T., Andò, E., Hughes, D. J. \& Viggiani, G. (2011). Can intergranular force transmission be identified in sand?. Gran. Matter 13, No. 3, 251-254.

Hurley, R., Marteau, E., Ravichandran, G. \& Andrade, J. E. (2014). Extracting inter-particle forces in opaque granular materials: beyond photoelasticity. J. Mech. Phys. Solids 63, No. 3, 251-254.

Hutchings, M. T., Withers, P. J., Holden, T. M. \& Lorentzen, T (2005). Introduction to the characterization of residual stress by neutron diffraction. Boca Raton, FL, USA: CRC Press Taylor \& Francis Group. 
Saadatfar, M., Sheppard, A. P., Senden, T. J. \& Kabla, A. J. (2012). Mapping forces in a 3D elastic assembly of grains. J. Mech. Phys. Solids 60, No. 1, 154-166.

Santisteban, J. R., Daymond, M. R., James, J. A. \& Edwards, L. (2006). ENGIN-X: a third-generation neutron strain scanner. J. Appl. Crystallogr. 39, No. 6, 812-825.

Stancik, A. L. \& Brauns, E. B. (2008). A simple asymmetric lineshape for fitting infrared absorption spectra. Vib. Spectrosc. 47, No. 1, 66-69.

Viggiani, G. \& Hall, S. A. (2008). Full-field measurements, a new tool for laboratory experimental geomechanics.
In Fourth Symposium on Deformation Characteristics of Geomaterials (eds E. B. Susan, W. M. Paul, J. C. Santamarina), vol. 1, pp. 3-26. Amsterdam, the Netherlands: IOS press.

Wakabayashi, T. (1950). Photo-elastic method for determination of stress in powdered mass. J. Phys. Soc. Jpn 5, No. 5, $383-385$.

Wensrich, C. M., Kisi, E. H., Zhang, J. F. \& Kirstein, O. (2012). Measurement and analysis of the stress distribution during die compaction using neutron diffraction. Gran. Matter 14, No. 6, 671-680.

\section{HOW CAN YOU CONTRIBUTE?}

To discuss this paper, please submit up to 500 words to the editor at journals@ice.org.uk. Your contribution will be forwarded to the author(s) for a reply and, if considered appropriate by the editorial board, it will be published as a discussion in a future issue of the journal. 